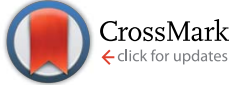

Cite this: RSC Adv., 2016, 6, 95101

Received 2nd March 2016

Accepted 13th September 2016

DOI: $10.1039 / c 6 r a 05548 f$

www.rsc.org/advances

\section{Timing of calcium nitrate addition affects morphology, dispersity and composition of bioactive glass nanoparticles $\dagger$}

\author{
Kai Zheng, ${ }^{\text {a }}$ Nicola Taccardi, ${ }^{\text {b }}$ Ana Maria Beltrán, ${ }^{\mathrm{c}}$ Baiyan Sui, ${ }^{\mathrm{d}}$ Tian Zhou, ${ }^{\mathrm{d}}$ \\ V. R. Reddy Marthala, ${ }^{e}$ Martin Hartmann ${ }^{e}$ and Aldo. R. Boccaccini ${ }^{\star a}$
}

Bioactive glass nanoparticles (BGN) are promising materials for a number of biomedical applications. Many parameters related to the synthesis of BGN using sol-gel methods can affect their characteristics. In this study, the influence of timing of calcium nitrate (calcium precursor) addition during processing on BGN characteristics was investigated. The results showed that the addition timing could affect the morphology, dispersity and composition of BGN. With delayed addition of calcium nitrate, larger, more regular and better dispersed BGN could be synthesized while the gap between nominal and actual compositions of BGN was widened. However, the addition timing had no significant influence on structural characteristics, as BGN with different addition-timing of calcium nitrate exhibited similar infrared spectra and amorphous nature. The results also suggested that monodispersed BGN could be synthesized by carefully controlling the addition of calcium nitrate. The synthesized monodispersed BGN could release Si and Ca ions continuously for up to at least 14 days. They also showed in vitro bioactivity and non-cytotoxicity towards rat bone marrow-derived mesenchymal stem cells (rBMSCs). In conclusion, the timing of calcium precursor addition is an essential parameter to be considered when producing BGN which should exhibit monodisperse characteristics for biomedical applications.

\section{Introduction}

Bioactive glasses (BG) are promising materials for hard tissue regeneration (e.g., bone and teeth), as they are bioactive, biocompatible, osteoconductive and osteoinductive. ${ }^{\mathbf{1 , 2}}$ In addition, BG can be fabricated conveniently in diverse morphologies, including three-dimensional scaffolds, ${ }^{3}$ fibers, ${ }^{4}$ coatings, ${ }^{5}$ and nanoparticles, ${ }^{6}$ for specific biomedical applications. Spherical bioactive glass nanoparticles (BGN) have started to attract increasing attention, ${ }^{7}$ due to their small size and large specific surface area which consequently lead to their high bioactivity and enhanced protein adsorption. ${ }^{7,8}$ Moreover, BGN are potential

\footnotetext{
${ }^{a}$ Institute of Biomaterials, Department of Materials Science and Engineering, University of Erlangen-Nuremberg, Cauerstrasse 6, 91058 Erlangen, Germany. E-mail: aldo.boccaccini@fau.de; Fax: +49 913185 28602; Tel: +49 91318528601 ${ }^{b}$ Institute of Chemical Reaction Engineering, University of Erlangen-Nuremberg, Egerlandstrasse 3, 91058 Erlangen, Germany

'Instituto de Ciencia de Materiales de Sevilla (CSIC-Universidad de Sevilla), 41092 Sevilla, Spain

${ }^{d}$ Shanghai Biomaterials Research \& Testing Center, Shanghai Key Laboratory of Stomatology, Ninth People's Hospital, Shanghai Jiaotong University, School of Medicine, 200023 Shanghai, China

${ }^{e}$ Erlangen Catalysis Resource Center, University of Erlangen-Nuremberg, Egerlandstrasse 3, 91058 Erlangen, Germany

$\dagger$ Electronic supplementary information (ESI) available. See DOI: 10.1039/c6ra05548f
}

materials as suitable carriers for delivering therapeutic ions ${ }^{9}$ or biomolecules. ${ }^{10}$ Additionally, BGN can be directly used as injectable materials for bone regeneration because of their unique flow properties, ${ }^{\mathbf{1 1 , 1 2}}$ and as inclusions in polymer biocomposites. ${ }^{\mathbf{1 3}}$

BGN can be synthesized by different methods, including flame spray synthesis ${ }^{6}$ and sol-gel methods. ${ }^{14}$ Sol-gel based methods are the most widely adopted way to make BGN due to the relatively low temperature processing and versatility., Aggregation is still one of the critical challenges for effective use of BGN, as aggregation may compromise the properties of BGN as building blocks for nanocomposites or injectable materials. ${ }^{1,7}$ A large number of investigations have been performed to improve the dispersity of sol-gel derived BGN. A combination of reverse micro-emulsion and sol-gel process could produce BGN with monodispersity. ${ }^{15}$ However, a high content of surfactants is needed to form inverse micelles and the resulting BGN are usually not homogeneous in size. Organic templates were also used to assist the synthesis of BG particles, but the dispersity and size homogeneity still need improvement. ${ }^{16-18}$ The Stöber method is convenient to produce monodispersed spherical silica-based nanoparticles in a range of 100-1000 nm. ${ }^{19-21}$ This method is performed under highly basic conditions, thus the formed nanoparticles could maintain dispersion due to the charge repulsion between the particles. ${ }^{22,23}$ However, the introduction of metallic ions in the course of BGN synthesis can change the surface charge of silica nanoparticles and may cause 
particles to coalesce and to aggregate. ${ }^{23}$ Based on the fact that the osteogenic property is mainly attributed to the release of ions upon BG exposure to biological fluids, ${ }^{24}$ the incorporation of metallic ions into BGN is necessary to achieve desired functionalities. In this context, the use of metallic ion precursors during BGN synthesis should be carefully controlled to achieve monodispersed BGN.

Calcium is a basic and important component of BG, as it contributes to the bonding to bone through the formation of hydroxyapatite (HA), ${ }^{25}$ and it enhances bone cell proliferation, differentiation, as well as the mineralization of the extracellular matrix, stimulating also the expression of growth factors. ${ }^{24,26}$ Recently, calcium alkoxide has been used as calcium precursor in the sol-gel synthesis of bioactive hybrid materials, ${ }^{\mathbf{1 , 2 7}}$ as calcium ions $\left(\mathrm{Ca}^{2+}\right)$ from this type of precursor could enter the silicate network at lower temperature compared with the $\mathrm{Ca}^{2+}$ ions from inorganic calcium salts. ${ }^{28}$ However, the relatively high cost and complicated use of calcium alkoxide still need to be considered. Calcium salts (usually calcium nitrate) are still the most widely adopted precursors for calcium in sol-gel processes. In a typical modified Stöber process for BGN synthesis, calcium nitrate is added after the formation of primary silica nanoparticles $(\mathrm{PSN})^{19,29}$ and $\mathrm{Ca}^{2+}$ ions enter the silicate network by diffusion during a period of high temperature treatment. ${ }^{28}$ However, the addition of salts may affect the stability of the $\mathrm{PSN},{ }^{30}$ which could cause irregular shape, ${ }^{29}$ inhomogeneity in size ${ }^{31}$ and aggregation..$^{32}$ It has been reported that the molar ratio of silica precursors to calcium nitrate is crucial to make monodispersed BGN and there is a threshold amount of calcium that can be incorporated into the BGN to achieve monodispersity. ${ }^{19}$ Moreover, the type of calcium salts may affect the final distribution of $\mathrm{Ca}^{2+}$ ions in the nanostructure of $\mathrm{BG}^{28}$

Larger particles usually have higher resistance to electrostatic interactions caused by the addition of electrolytes including calcium salts. ${ }^{23}$ Considering the continuous growth of particles during the reaction in the Stöber process, we hypothesized that the timing of the addition of calcium nitrate could affect the characteristics of the resulting material. If other process parameters are controlled, adding calcium nitrate in the late stages of the Stöber process may avoid the aggregation and irregularity of particles. One of the aims in this study was thus to investigate the influence of timing of calcium nitrate addition on the characteristics of the produced BGN. Calcium nitrate was added after formation of PSN at different times. The influence of this addition-timing difference on the morphology, composition and structure of BGN was investigated and discussed. In addition, an optimized facile and rapid routine for synthesizing monodispersed BGN was developed based on the experimental results. To evaluate the possible biomedical applications of synthesized monodispersed BGN, their ion release behavior, bioactivity and cytotoxicity were also investigated.

\section{Experimental sections}

\subsection{Synthesis of BGN}

BGN were synthesized by a modified Stöber method (see Fig. 1). The nominal composition of $\mathrm{BGN}$ was $59.4 \mathrm{SiO}_{2}-36.5 \mathrm{CaO}-$
$4.1 \mathrm{P}_{2} \mathrm{O}_{5}$ (in mol\%). Briefly, solution A was prepared by dissolving $2.25 \mathrm{~mL}$ of tetraethyl orthosilicate (TEOS, 98\%, Sigma-Aldrich) and $0.23 \mathrm{~mL}$ of triethyl phosphate (TEP, 99.8\%, Sigma-Aldrich) in $25 \mathrm{~mL}$ of ethanol (96\% VWR). Solution B was prepared by mixing $4.5 \mathrm{~mL}$ of ammonium hydroxide solution (28.0-30.0\%, Sigma-Aldrich), $8.12 \mathrm{~mL}$ of ethanol and $12.38 \mathrm{~mL}$ of deionized water. Solution A was then poured onto solution B rapidly under continuous stirring. After leaving the reaction to proceed for a pre-determined time $(0,10,30$ and 60 minutes, respectively), $1.45 \mathrm{~g}$ of calcium nitrate (99\%, Sigma-Aldrich) was added to the mixture. Finally, the mixtures were allowed to react for a further period of time (total time after the mixing of solution A and B was $2 \mathrm{~h}$ ). For BGN-60, the reaction time was set as 2 hours and a half to ensure that BGN-30 and BGN-60 would have the same time available for the interactions between silica and calcium precursors to occur. The resulting whitish suspensions were centrifuged at $7830 \mathrm{rpm}$ (Centrifuge 5430R, Eppendorf) for $5 \mathrm{~min}$ to obtain deposits and they were further washed twice using deionized water and once using ethanol. The collected deposits were dried at $60{ }^{\circ} \mathrm{C}$ for $6 \mathrm{~h}$ before being calcined at $700{ }^{\circ} \mathrm{C}$ for $2 \mathrm{~h}$. The heating rate was $2{ }^{\circ} \mathrm{C} \min ^{-1}$ and the material was then cooled down overnight in the furnace. The obtained BGN were labelled correspondingly BGN-0, BGN-10, BGN-30 and BGN-60, respectively. In the case of BGN-0, calcium nitrate was dissolved in solution A before mixing.

\subsection{Characterization of $\mathbf{B G N}$}

The synthesized BGN were ultrasonically dispersed in ethanol before being dropped directly on a sample stub for SEM observation. After drying, the BGN were observed by field emission scanning electron microscopy (FE-SEM; Auriga, Carl Zeiss) without being sputter-coated. SEM images were analyzed using Image (NIH, USA) for the determination of particle size. The structure of BGN was also assessed by high resolution transmission electron microscopy (HRTEM) using a FEI Tecnai G2F30 S-Twin microscope operated at $300 \mathrm{kV}$ and equipped with a HAADF Fischione detector. For the TEM observation, particles were homogeneously dispersed in ethanol by ultrasound and then dropped on a carbon film.

Zeta-potential measurements were conducted using a Zetasizer nano ZS (Malvern Instruments, UK) to determine the surface charge of BGN. For zeta-potential analysis, BGN were suspended in deionized water at the concentration of $0.5 \mathrm{~g} \mathrm{~L}^{-1}$.

Fourier transform infrared spectroscopy (FTIR) spectra were recorded in transmission mode using a Nicolet 6700 FTIR spectrophotometer (Thermo Scientific, USA) under ambient conditions. The weight ratio of sample to $\mathrm{KBr}$ was set at $1: 100$. The spectra were collected between 2400 and $400 \mathrm{~cm}^{-1}$ with the resolution of $4 \mathrm{~cm}^{-1}$.

$\mathrm{X}$-ray diffraction (XRD) analysis was performed using a D8 ADVANCE X-ray diffractometer (Bruker, USA) in a $2 \theta$ range of $20-80^{\circ}$ with $\mathrm{Cu} \mathrm{K} \alpha$ radiation. All BGN were dispersed in ethanol before being deposited on low-background silicon wafers (Bruker AXS). A step size of $0.014^{\circ}$ with a dwell time of $1 \mathrm{~s}$ per step was used. 


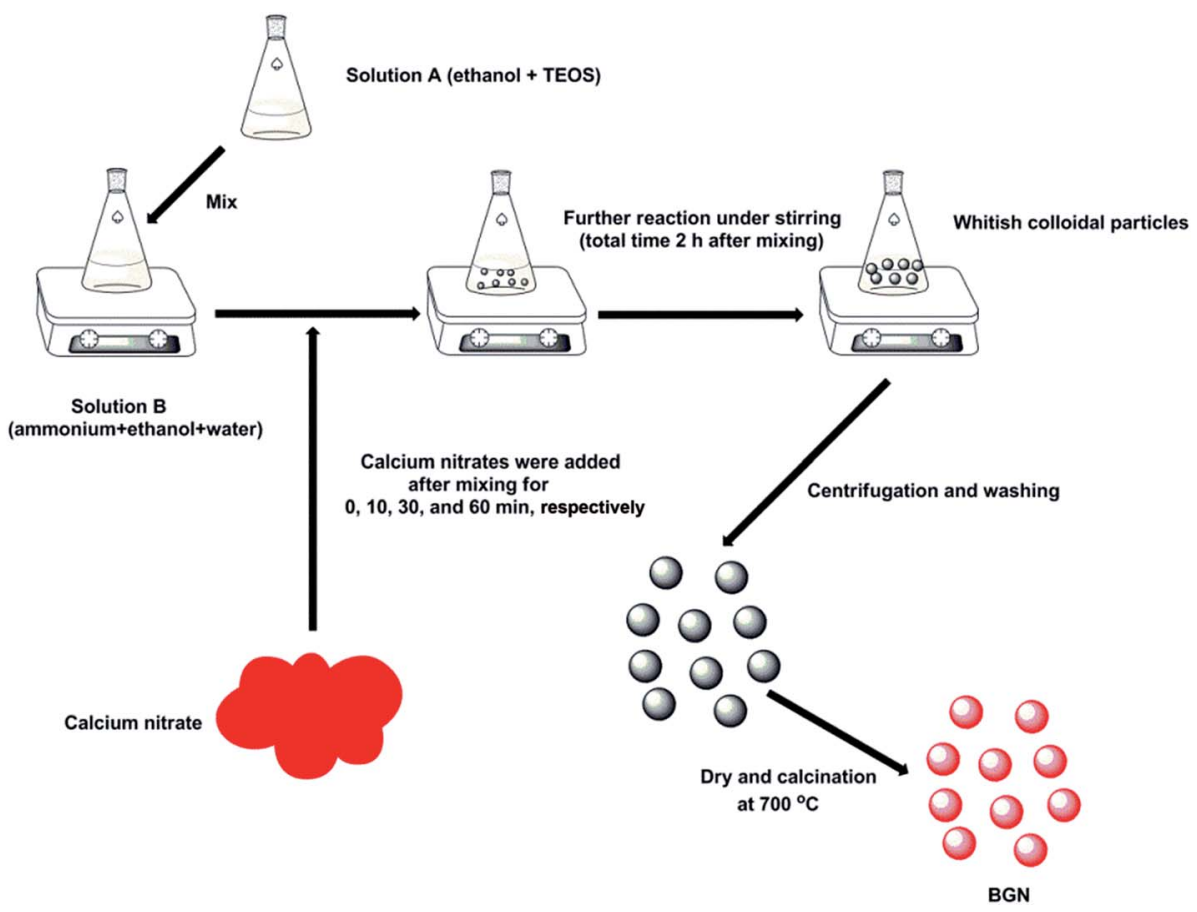

Fig. 1 Schematic representation showing the synthesis route for BGN with different addition-timing of calcium nitrate.

To analyze the elemental composition of BGN, $50 \mathrm{mg}$ of sample for each type of BGN was digested for inductively coupled plasma atomic emission spectroscopy (ICP-AES, SPECTRO CIROS-CCP spectrometer) measurements. The digestion was conducted using microwave heating (the heating program was: room temperature to $180^{\circ} \mathrm{C}$ in $5 \mathrm{~min}$ and held for $5 \mathrm{~min}$, then a second ramp up to $230^{\circ} \mathrm{C}$ in $5 \mathrm{~min}$ and held for 20 $\mathrm{min}$, followed by cooling down at room temperature). $10 \mathrm{~mL}$ of concentrated $\mathrm{HF} / \mathrm{HNO}_{3} / \mathrm{HCL}$ mixture in $1 / 1 / 3$ ratio was used as digestion medium. The resulting samples were diluted to 100 $\mathrm{mL}$ with deionized water for the analysis.

One-pulse ${ }^{29} \mathrm{Si}$ HPDEC (high power hetero-nuclear dipolar decoupling) MAS NMR measurements were performed on an Agilent DD2 $500 \mathrm{MHz}$ WB spectrometer at a resonance frequency of $99.30 \mathrm{MHz}$. The ${ }^{29} \mathrm{Si}$ spectra were recorded using a $6 \mathrm{~mm}$ MAS NMR probe with a sample spinning rate of $5 \mathrm{kHz}$ and by applying a pulse length of $3.9 \mu$ s and a relaxation delay of $60 \mathrm{~s}$ for BGN-30 and calcium-free silica particles (without addition of calcium nitrate). Approximately 1200 scans were accumulated for BGN-30, while 4000 scans were accumulated for the calcium-free silica sample.

\subsection{Degradation behavior in DMEM}

The assessment of the degradation rate of BGN-30 was performed in low-glucose Dulbecco's Modified Eagle Medium (LDMEM; Sigma-Aldrich, Germany). BGN-30 were selected as they were monodispersed and homogenous in size. In addition, BGN-30 exhibited relatively high calcium content compared with BGN-60. Briefly, $75 \mathrm{mg}$ of BGN-30 was immersed in $50 \mathrm{~mL}$ of DMEM at $37{ }^{\circ} \mathrm{C}$ and $120 \mathrm{rpm}$. At each pre-determined time point, $0.5 \mathrm{~mL}$ of supernatant liquid was extracted from the medium and the same amount of fresh DMEM was replenished.
The extracts were then diluted to $10 \mathrm{~mL}$ with deionized water for ICP measurements. DMEM without BGN-30 was measured and the obtained values were used as background.

\subsection{In vitro mineralization}

The apatite-forming ability of monodispersed BGN was evaluated as described in previous reports. ${ }^{17,35}$ Briefly, BGN-30 particles were immersed in simulated body fluid (SBF) in an incubator (KS 4000i control, IKA, Germany) at $37{ }^{\circ} \mathrm{C}$ and 120 rpm for up to 7 days. SBF was replaced twice per week to keep the ion concentration stable. At every pre-determined time point, BGN-30 were removed from the SBF and rinsed with deionized water. The collected samples were then dried at $60^{\circ} \mathrm{C}$ for $24 \mathrm{~h}$ before being characterized by FE-SEM and FTIR. The procedures of characterization were the same as described above.

\subsection{Cell viability assays}

The experimental protocol was approved by the Animal Care and Experiment Committee of Ninth People's Hospital affiliated to School of Medicine, Shanghai Jiao Tong University. The experiments were conducted following Shanghai Administration Rule of Laboratory Animals. Rat bone marrow-derived mesenchymal stem cells (rBMSCs) were obtained from the femora of 4 week-old Sprague-Dawley rats. In brief, the marrow of femora midshaft was flushed out and suspended in L-DMEM (Hyclone, USA) supplemented with $10 \%$ fetal bovine serum (FBS, Hyclone), $100 \mathrm{U} \mathrm{mL}^{-1}$ penicillin and $100 \mathrm{mg} \mathrm{L}^{-1}$ streptomycin (Hyclone). Non-adherent cells were removed after 3 days. When reached approximately $80 \%$ confluence, the cells 
were passaged and used for the following experiment from second to third passages.

To evaluate the in vitro cytotoxicity of monodispersed BGN, extracts from BGN-30 were prepared. Briefly, the samples were incubated for $24 \mathrm{~h}$ at $37^{\circ} \mathrm{C}$ in L-DMEM at a ratio of $1 \mathrm{mg} \mathrm{mL}{ }^{-1}$. The mixture was then centrifuged and the supernatant was collected. Serial dilutions $\left(500,250\right.$, and $\left.125 \mu \mathrm{g} \mathrm{mL}{ }^{-1}\right)$ of extracts were prepared using L-DMEM medium for further cell culture experiments. The medium supplemented without addition of material extracts was used as control. Samples were sterilized by gamma radiation at $25 \mathrm{KGY}$ before use.

Cell viability was evaluated by the 3-(4,5-dimethylthiazol-2yl)-2,5-diphenyl tetrazolium bromide (MTT) colorimetric assay. Briefly, rBMSCs were seeded in 96-well plates at $3.1 \times 10^{4}$ cells per $\mathrm{cm}^{2}$. The obtained extracts were added respectively and incubated for $24 \mathrm{~h}$. The MTT assay was performed following the manufacturer's instructions. Dimethyl sulfoxide (DMSO, SigmaAldrich) was used to dissolve the formazan crystals, and the optical density was measured at $570 \mathrm{~nm}$ using a microplate reader (Multiskan ${ }^{\mathrm{TM}}$ FC, Thermo Fisher Scientific, USA).

\subsection{Statistical analysis}

All quantitative experiments were carried out at least in triplicate, and data were shown as mean \pm standard deviation (S.D.). Statistical analyses were performed using SPSS software (v 12.0; IBM Corporation, NY, USA), and statistical comparisons were analyzed using the $t$-test. Differences were considered statistically significant when $P<0.05$.

\section{Results and discussion}

\subsection{Influence on morphology}

Fig. 2 shows SEM images of BGN-0, BGN-10, BGN-30 and BGN60 . As can be seen, the morphology of BGN varied significantly with the change of addition-timing of calcium nitrate. BGN0 exhibited the smallest size $(<100 \mathrm{~nm})$ among the BGN, but they considerably aggregated. BGN-10 showed a dual-size distribution, in which small particles $(<100 \mathrm{~nm})$ and large particles $(\sim 400 \mathrm{~nm})$ co-existed in the manner that small particles clustered around the larger ones. As a comparison, both BGN-30 and BGN-60 were monodispersed and showed similar spherical shape with homogenous size $(\sim 400 \mathrm{~nm})$.

TEM images of the samples exhibited the typical morphological features of BGN (Fig. 3). BGN-0 were composed of aggregated small particles (Fig. 3a), and voids between particles could be observed in the TEM image. BGN-10 exhibited morphology of smaller particles clustering on larger particles (Fig. 3b), and voids between large and small particles could be observed. BGN-30 and BGN-60 exhibited monodispersed and spherical morphology (Fig. 3c and d), as observed in SEM images. Additionally, no obvious nanopores could be observed in all BGN, which is in agreement with results of previous studies reporting that particles produced via the Stöber method were non-porous after calcination at high temperature. ${ }^{\mathbf{1 9 , 2 0 , 2 9}}$ The above results clearly confirm that the morphology of BGN can be affected by the addition-timing of calcium nitrate. Furthermore, it was shown in this study that monodispersed BGN with spherical shape could be synthesized after adding

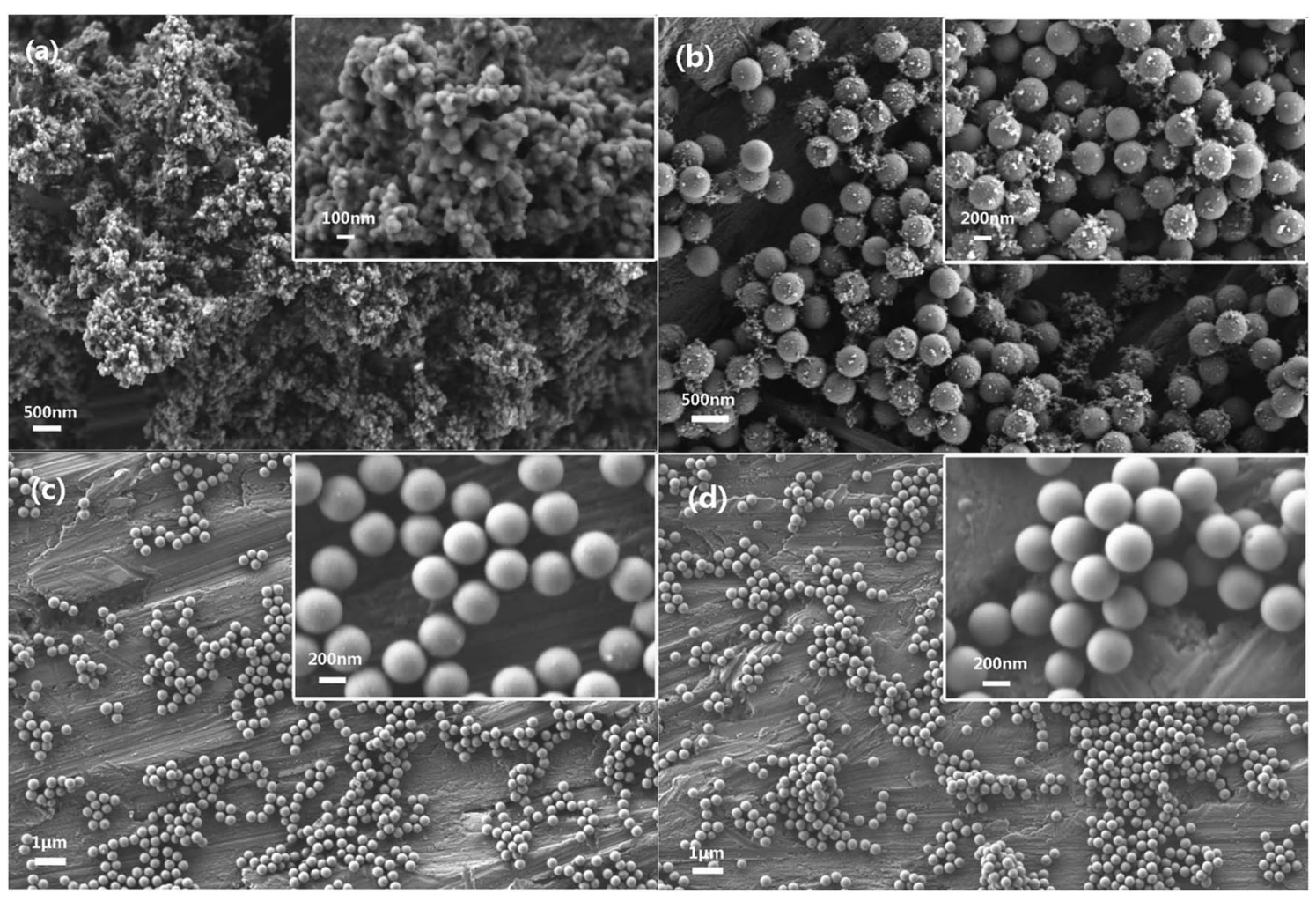

Fig. 2 SEM images of (a) BGN-0, (b) BGN-10, (c) BGN-30, and (d) BGN-60 at low magnification and (inserted) high magnification. 


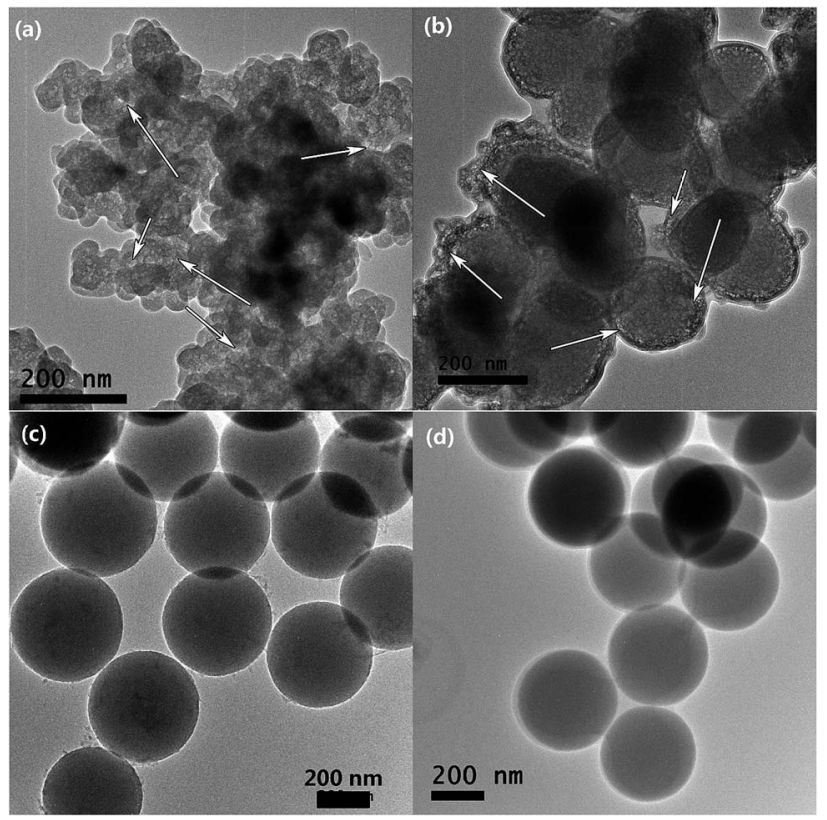

Fig. 3 TEM images of (a) BGN-0, (b) BGN-10, (c) BGN-30, and (d) $B G N-60$. Arrows indicate the voids between particles.

calcium nitrate at certain time points $(>30$ min after mixing solution A and B).

The influence of addition-timing of calcium nitrate on BGN morphology could be explained by the stability mechanism of silica particles in electrolytic solution. ${ }^{30}$ In a typical Stöber process, PSN form within seconds or minutes after the mix of
TEOS and catalyst. ${ }^{23}$ The PSN then continue growing by Ostwald ripening and form spherical secondary particles. ${ }^{1}$ These charged secondary particles can remain stable in particulate morphology without being bonded because they mutually repel each other in highly basic conditions. ${ }^{23}$ However, the existence of $\mathrm{Ca}^{2+}$ ions may lead to the aggregation of silica particles due to electrostatic interactions between the hydrated silica surface and hydrated counterions. ${ }^{30}$ To better understand the influence of addition timing, the formation process of BGN is schematically illustrated in Fig. 4 . In the case of BGN-0, a large number of $\mathrm{Ca}^{2+}$ ions was present before the formation of primary particles. $\mathrm{The}^{\mathrm{Ca}^{2+}}$ ions could then be adsorbed on the surface of PSN as soon as the particle formation took place, which caused the instability and aggregation of the particles due to electrostatic interactions. ${ }^{30}$ Consequently, BGN-0 exhibited a gel-like structure composed of bonded small particles (Fig. 3a). In the case of BGN-10, part of the particles has been able to grow to a relative large extent to withstand the effect induced by $\mathrm{Ca}^{2+}$ ions. However, some smaller particles could still be influenced by $\mathrm{Ca}^{2+}$ ions and they are then adsorbed by the larger particles. This can probably explain the co-existence of large and small particles in BGN-10 sample. Notably, almost all particles might grow to a relatively large size after reaction for $30 \mathrm{~min}$, suggesting that electrostatic forces were insufficient to withstand the mechanical strain caused by Brownian motion. ${ }^{23}$ As a result, BGN-30 and BGN-60 exhibited monodispersed and spherical morphology (Fig. 2c and d) with homogenous distribution of particle size. In addition, the zeta-potential of BGN-0, BGN-10, BGN-30 and BGN-60 were $\sim-21, \sim-21, \sim-25$, and $\sim-23 \mathrm{mV}$,

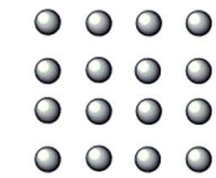

$0 \mathrm{~min}$
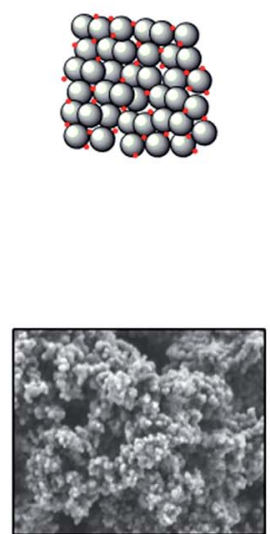

BGN-0

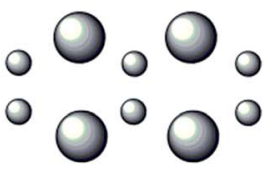

$10 \mathrm{~min}$
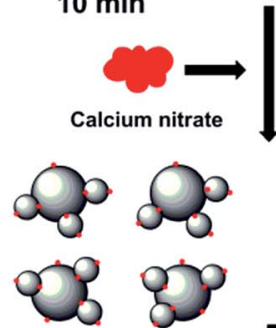

Calcination

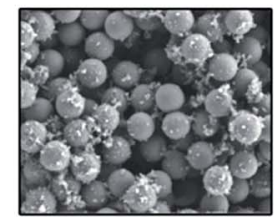

BGN-10

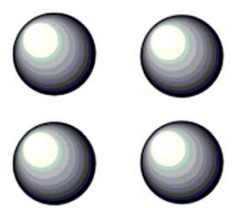

$30 \mathrm{~min}$
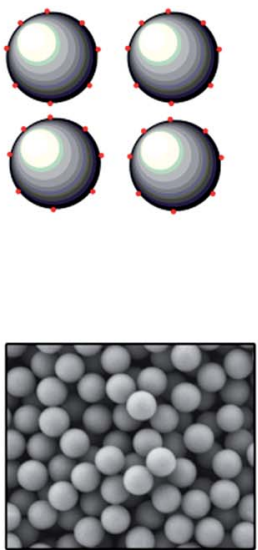

BGN-30

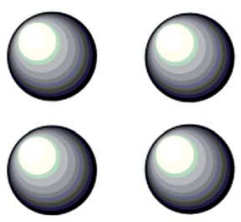

$60 \mathrm{~min}$
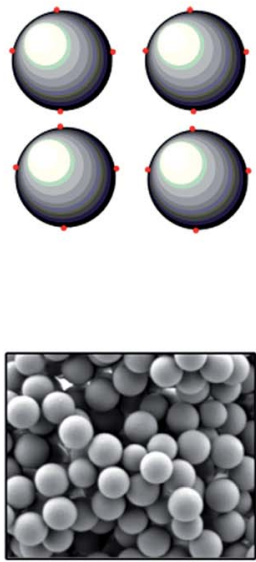

BGN-60

Fig. 4 Schematic illustration demonstrating the formation process of BGN with different addition-timing of calcium nitrate at minute $0,10,30$, and 60 after mixing solution $A$ and $B$. 
Table 1 Comparison of the actual compositions of different BGN determined by ICP-AES. The nominal composition of BGN is $59.4 \mathrm{SiO}_{2}-36.5 \mathrm{CaO}-4.1 \mathrm{P}_{2} \mathrm{O}_{5}$ by mol\% (N.D.: not detectable)

\begin{tabular}{llrl}
\hline Glass type & $\mathrm{SiO}_{2}(\mathrm{~mol} \%)$ & $\mathrm{CaO}(\mathrm{mol} \%)$ & $\mathrm{P}_{2} \mathrm{O}_{5}(\mathrm{~mol} \%)$ \\
\hline BGN-0 & $67.1 \pm 1.2$ & $32.9 \pm 0.3$ & N.D. \\
BGN-10 & $80.9 \pm 2.7$ & $19.1 \pm 1.6$ & N.D. \\
BGN-30 & $91.1 \pm 0.5$ & $8.9 \pm 1.4$ & N.D. \\
BGN-60 & $97.2 \pm 0.6$ & $2.8 \pm 0.3$ & N.D.
\end{tabular}

respectively, indicating that all BGN suspensions should be stable enough for preparing nanocomposites or coatings and confirming the negatively surface charge of BGN.

\subsection{Influence on composition}

Table 1 summarizes the nominal and actual compositions of synthesized BGN. It is notable that phosphorous (P) was not detected by ICP-AES measurements, suggesting that only limited amount of $\mathrm{P}$ was incorporated in BGN (below the detection limit of ICP measurements). Because $\mathrm{P}$ is considered to facilitate apatite formation on BGN after exposure to body fluid, a nominal composition containing $\mathrm{P}$ was designed for BGN in this study. Although previous studies have revealed that P-containing BGN could be prepared using sol-gel methods, this type of glasses has been rarely reported using the Stöber method. The results indicate that $\mathrm{P}$ was rarely or not incorporated into BGN using the modified Stöber method, and the influence of the addition timing of calcium nitrate on the incorporation of $\mathrm{P}$ into BGN was not significant. This result is consistent with results of previous studies, ${ }^{15,20,28,29}$ which could be explained by the different hydrolysis rates between TEOS and TEP as well as the favorable watersolubility of TEP. As discussed above, TEOS can be hydrolyzed and condensed within seconds or minutes under strong basic conditions, while the hydrolysis rate of TEP is relatively slow in a basic environment. This unbalanced hydrolysis rate could thus cause the formation of silica particles before hydrolysis of TEP, so that limited or no $\mathrm{P}$ species could enter the silicate network. Additionally, the remaining TEP could be removed in the course of the following washing process due to its high water-solubility, which excluded the possibility of incorporating $\mathrm{P}$ by diffusion during the subsequent heat treatment. In order to synthesize P-containing BGN using the Stöber method, further studies focused on optimizing the hydrolysis of $\mathrm{Si}$ and $\mathrm{P}$ precursors are still needed.

Moreover, it should be noted that there was a lower concentration of incorporated calcium in BGN compared with the nominal composition. It is known that a gap exists between the nominal and actual compositions of sol-gel derived BGN. ${ }^{19,20}$ The centrifugation and washing processes before drying are usually considered to be plausible reasons leading to the composition gap, ${ }^{19}$ because un-reacted precursors can be found in the removed solutions. The removal of these excess unreacted precursors is necessary, since excess TEOS and ammonium may cause further undesired reactions during the drying process and excess calcium nitrates in sols may form calcium hydroxide instead of being incorporated into the silica particles. ${ }^{33}$ However, the centrifugation and washing processes were not the main factors affecting composition difference in this study.

As can be seen in Table 1, different BGN had significantly different compositions, but they had undergone the same centrifugation and washing processes. The addition-timing of calcium nitrate was thus the main factor affecting the composition of BGN. The incorporated amount of calcium in BGN decreased with the delay of calcium addition. The composition of BGN-0 was the most similar to the nominal composition. Only $\sim 2.8$ and $\sim 8.9 \mathrm{~mol} \%$ of $\mathrm{CaO}$ were incorporated in BGN-60 and $\mathrm{BGN}-30$, while $\sim 19.1$ and $36.5 \mathrm{~mol} \%$ of $\mathrm{CaO}$ could be incorporated in BGN-10 and BGN-0, respectively. This difference could be clarified by observing the formation process of BGN (Fig. 4). $\mathrm{Ca}^{2+}$ ions could be adsorbed on the surface of silica particles due to the electrostatic interactions between positively charged $\mathrm{Ca}^{2+}$ ions and negatively charged $\mathrm{Si}-\mathrm{OH}$ groups. They could also form hydrated calcium silicate with $\left[\mathrm{SiO}_{4}\right]^{4-}$ groups and (or) calcium hydroxide with $\mathrm{OH}$ - groups. ${ }^{30,34}$ Hence, it is understandable that the particles with more $\left[\mathrm{SiO}_{4}\right]^{4-}$ and $\mathrm{OH}_{-}$ groups could adsorb more $\mathrm{Ca}^{2+}$ ions. As mentioned above, PSN form quickly and grow subsequently by Ostwald ripening under strong basic conditions. While the particles grow, the number of particles decreases over the whole process of synthesis. Obviously, the system BGN-0 had the largest number of particles with the smallest size. As a result, larger amounts of $\left[\mathrm{SiO}_{4}\right]^{4-}$ and $\mathrm{OH}-$ groups were available in BGN-0 compared with the other synthesis systems. The particles in BGN-0 system could then adsorb the largest number of $\mathrm{Ca}^{2+}$ ions among all systems. Moreover, the particles in BGN-0 system had lost stability and aggregated at an early stage of reaction, in which $\mathrm{Ca}^{2+}$ ions could be "locked" inside the structure being relatively difficult to lose them in the following washing step. The above two explanations could therefore account for the highest concentration of calcium in BGN-0. Actually, the situation of BGN-0 was similar to acid-catalyzed sol-gel BG (the addition of calcium nitrate before mixing also reduced the $\mathrm{pH}$ value in the medium), in which the PSN are aggregated to form a gel structure. ${ }^{28}$ Therefore, the composition of acid-catalyzed BG is usually close to the designed composition, considering the absence of a washing process. ${ }^{35}$ In the case of monodispersed BGN-30 and BGN-60, secondary silica particles had grown to a relatively large size when the addition of the calcium precursor took place, which means that fewer sites and pores were available for adsorption of $\mathrm{Ca}^{2+}$ ions. Consequently, the particles in $\mathrm{BGN}-30$ and $\mathrm{BGN}-60$ could only incorporate a limited calcium content. The relatively low amount of calcium in BGN-60 in comparison to BGN-30 is understandable, considering the larger particle size of BGN-60 during the addition of calcium nitrate. It should be noted that the reaction time between silica and calcium precursors was different for different BGN, which may also lead to the different amount of calcium incorporated. However, the influence of reaction time in this study could be limited, as the interaction time between silica and calcium precursors in BGN-30 and BGN-60 was the same while the composition gap was still significant. Therefore, 
the BGN composition is expected to be mainly influenced by the addition-timing of calcium nitrate.

In summary, the addition timing of Ca precursor could affect the actual composition of BGN, which was determined by the mechanism of particle formation in the Stöber process. With delayed addition of calcium nitrate, the gap between nominal and actual compositions of BGN could be widened. Furthermore, calcium precursor concentrations are likely over saturated for the synthesis of BGN-30 and BGN-60, which means that it is possible to make monodispersed BGN with similar composition to BGN-30 by using a lower amount of calcium precursor. Additionally, the centrifugation and washing processes may also affect the composition of BGN by removing un-reacted precursors, but this influence was not significant. It has been reported that more calcium could be incorporated by the careful control of $\mathrm{pH},{ }^{36,37}$ the use of aerosol techniques ${ }^{38}$ or the combination of polymer templates. ${ }^{39}$ However, the compositional results in the above reports were obtained only by energy-dispersive X-ray spectroscopy. Moreover, complex synthesis processes or equipment is needed in the suggested preparation protocols. Therefore, the optimization of the Stöber method for producing monodispersed BGN with high incorporated amount of $\mathrm{Ca}$ is needed in further studies. Nevertheless, the actual composition of BGN could be controlled by carefully tuning relevant processing parameters (e.g. addition timing of precursors and washing process) in the Stöber method.

\subsection{Influence on structure}

Fig. 5a shows the FTIR spectra of BGN-0, BGN-10, BGN-30 and BGN-60. A broad band starting at $460 \mathrm{~cm}^{-1}$ can be assigned to the $\mathrm{Si}-\mathrm{O}-\mathrm{Si}$ rocking mode, while a band located at $812 \mathrm{~cm}^{-1}$ can be assigned to the $\mathrm{Si}-\mathrm{O}-\mathrm{Si}$ symmetric stretching mode. ${ }^{35}$ All the above bands are characteristic bands of silicate glasses. Additionally, a strong band located at around $1630 \mathrm{~cm}^{-1}$ can be attributed to the vibration of hydroxyl groups, which indicates that a large content of water was available on the sample surface. ${ }^{40}$ Notably, two bands at 1412 and $1498 \mathrm{~cm}^{-1}$, which can respectively be assigned to the stretching vibration of $\mathrm{C}-\mathrm{O}$ bond and $\mathrm{O}-\mathrm{H}$ bending vibration bond, ${ }^{41}$ are only observed in the FTIR spectra of BGN-0 and BGN-10. This result could be explained by the higher content of $\mathrm{Ca}^{2+}$ ions and larger surface area of BGN-0 and BGN-10 leading to the formation of calcium carbonate. ${ }^{28}$ Furthermore, a band at $1874 \mathrm{~cm}^{-1}$, which is attributed to the $\mathrm{Si}-\mathrm{O}-\mathrm{Si}$ stretching vibration in highly condensed silicate materials, can only be seen in the spectra of BGN-30 and BGN-60. This phenomenon indicates that higher levels of poly-condensation in BGN-30 and BGN-60 were available compared with BGN-0 and BGN-10. ${ }^{42}$

The XRD patterns of BGN (Fig. 5b) showed a broad band in the range of $2 \theta=\sim 20-35^{\circ}$, which is a typical characteristic peak for amorphous silicate materials ${ }^{43}$ and its existence confirms the amorphous nature of all synthesized BGN. Notably, the broad band of BGN-0 exhibited slight shift compared with other BGN. This could be attributed to amorphous calcium silicate species. ${ }^{44}$ All different types of BGN exhibited similar FTIR spectra and XRD patterns, indicating that the timing of addition of calcium precursor exerted negligible influence on the intrinsic structure of the BGN.

\subsection{Properties of monodispersed BGN}

On the basis of the above discussion, it can be concluded that the timing of addition of calcium nitrate affects the morphology, dispersity and composition of BGN. Moreover, monodispersed spherical BGN could be synthesized by carefully controlling the addition-timing of calcium nitrate. In this study, monodispersed BGN could be created by adding calcium nitrate 30 min later after mixing solution $\mathrm{A}$ and $\mathrm{B}$. This monodispersity should be beneficial for the effective applications of BGN in nanocomposites, bioactive coatings or injectable materials. To evaluate the potential of monodispersed BGN in biomedical applications, the bioactivity, ion release behavior, and cytotoxicity of BGN were assessed. BGN-30 was selected to evaluate the properties of monodispersed BGN, as it contains more calcium than BGN-60.

3.4.1. In vitro bioactivity. Fig. 6a shows the FTIR spectra of BGN-30 upon immersion in SBF for 3 and 7 days. It can be seen that two new bands at 564 and $604 \mathrm{~cm}^{-1}$ appeared after 3 days of immersion in SBF. These two bands can be assigned to $\mathrm{P}-\mathrm{O}$ bonds in crystallized apatite and usually indicate the formation of HA in BG. ${ }^{35}$ Additionally, two new bands at 1417 and 1458 $\mathrm{cm}^{-1}$, being assigned to the stretching vibration of $\mathrm{C}-\mathrm{O}$
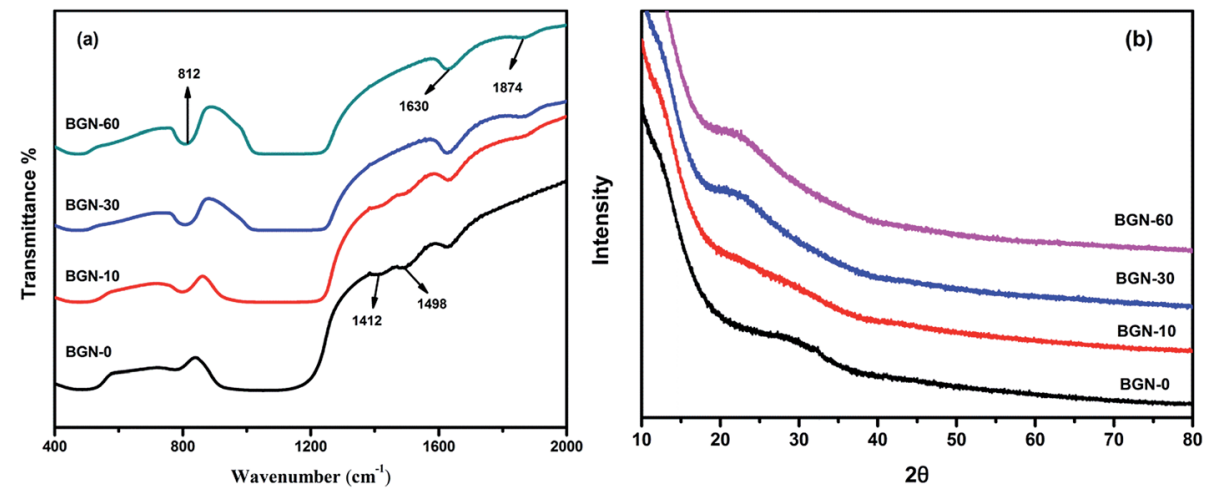

Fig. 5 Structural characteristics of BGN-0, BGN-10, BGN-30 and BGN-60, respectively. (a) FTIR spectra; (b) XRD patterns. 


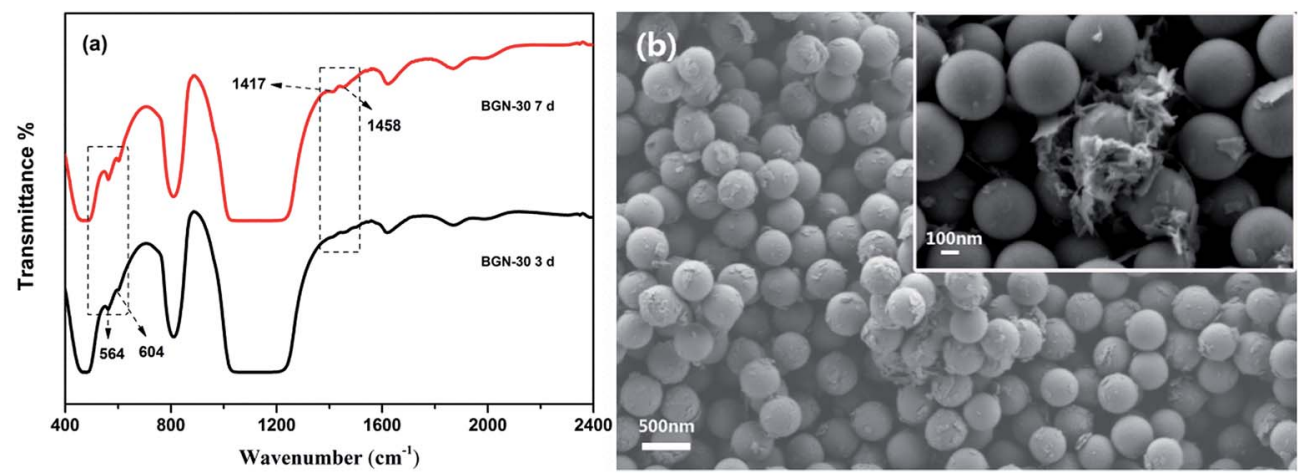

Fig. 6 Bioactivity evaluation of monodispersed BGN-30. (a) FTIR spectra of BGN-30 after immersion in SBF for up to 7 days; (b) SME images showing HCA crystals on BGN-30 after 3 days of immersion in SBF and higher magnification SEM image (insert).

bonds,$^{45}$ can be seen in the spectra. This result suggests that carbonated hydroxyapatite (HCA) rather than stoichiometric HA formed on BGN-30 after immersion in SBF for 3 days. The intensity of these characteristic bands increased after 7 days of immersion, which indicates that more crystallized HA has formed. Fig. $6 \mathrm{~b}$ shows the SEM image of BGN-30 after 3 days of immersion in SBF. It can be seen that needle-like crystals, which have the typical morphology of HCA formation on nanoscale $\mathrm{BG},{ }^{46}$ clustered on the BGN-30. These results therefore indicate that monodispersed BGN-30 are bioactive and HCA could form on BGN-30 in a short-period (3 days) of immersion in SBF. However, it should be pointed out that calcium content of BGN$30(\sim 8.9 \%)$ was lower than the limit of calcium content considered to impart bioactivity $(\sim 10 \%)$ in sol-gel derived BG. ${ }^{47}$ This apparent paradox could be explained in term of the high local calcium content (maybe $>10 \%$ ) near the surface of BGN-30. Bioactivity assessment in previous studies has shown similar HA formation on low calcium content doped silica nanoparticles. ${ }^{48,49}$

3.4.2. Degradation behavior in DMEM. DMEM is usually used as a mimetic cell culture environment to assess the degradability of materials, since dissolution products in DMEM could be utilized directly to evaluate the biocompatibility and osteogenic activity of materials. ${ }^{50}$ However, DMEM intrinsically contains abundant levels of potassium, calcium, and amino acids, leading to possible interaction with the tested material over time. ${ }^{51}$ In this study, the assessment medium (DMEMWater mixture) contained $\mathrm{Ca}^{2+}$ ions at a concentration of $\sim 6.5$ $\mathrm{mg} \mathrm{mL}^{-1}$. It is notable that $\mathrm{Ca}^{2+}$ ions were released from BGN30 during the first 1 hour immersion while the calcium concentration in the medium decreased in the following 3 days of immersion (Fig. 7a). This phenomenon could be due to the interaction between BGN and DMEM causing an amorphous Ca-P layer formation on the surface of the particles, ${ }^{51}$ which consumed the $\mathrm{Ca}^{2+}$ ions in the medium. With the increase of immersion time, the formation of a Ca-P layer is likely to slow down and the release of $\mathrm{Ca}^{2+}$ ions from BGN-30 will be higher than the consumption of $\mathrm{Ca}^{2+}$ ions from the medium. This effect could account for the increase of $\mathrm{Ca}^{2+}$ ions after immersion in DMEM for 3 days. In addition, more $\mathrm{Ca}^{2+}$ ions were released from BGN-30 in the following immersion period. This stable release of $\mathrm{Ca}^{2+}$ ions for up to 14 days in DMEM indicates that calcium should be incorporated into the silicate structure of BGN instead of being only adsorbed on the surface of the particles. The physically adsorbed $\mathrm{Ca}^{2+}$ ions would only exhibit a burst release in a short time, however, this stable release of $\mathrm{Ca}^{2+}$ ions for up to 14 days could result from the degradation of glasses. BGN-30 also showed a stable Si release for up to 14 days in DMEM, which suggests that BGN-30 was degradable in DMEM.

The continuous ion-release feature is significant considering the expected positive biological response triggered by $\mathrm{BGN}^{24}$
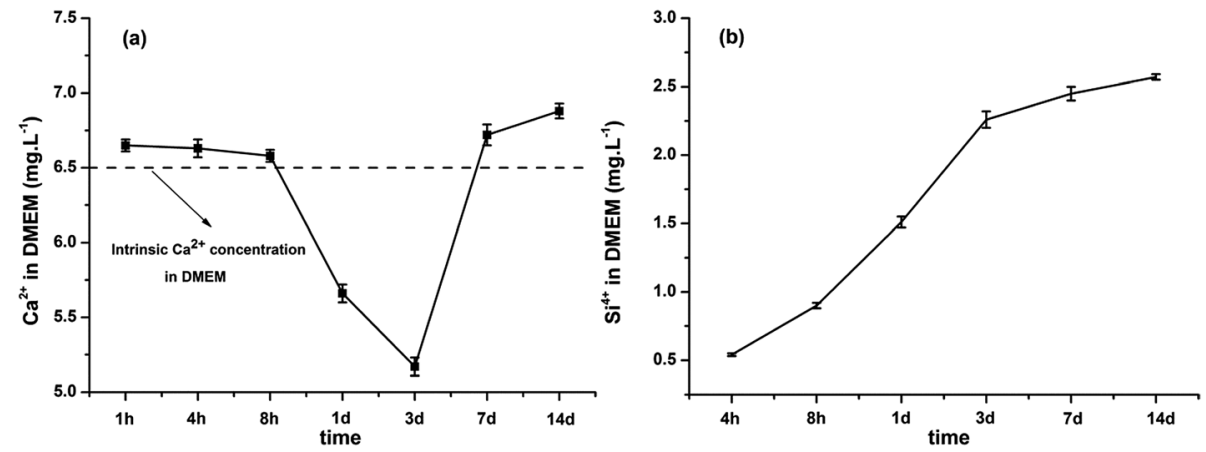

Fig. 7 Ion release behavior of BGN-30 in DMEM solution. (a) Ca and (b) Si ions released from the BGN-30 for up to 14 days. 

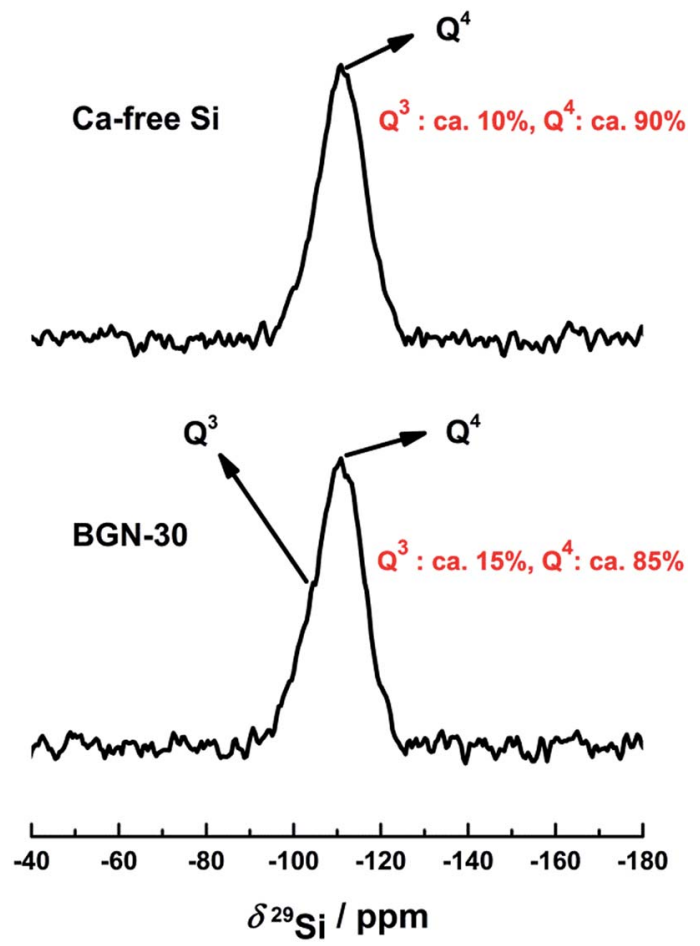

Fig. $8{ }^{29} \mathrm{Si}$ HPDEC MAS NMR spectra of BGN-30 and Ca-free silica nanoparticles. The relative intensities of $Q^{3}$ and $Q^{4}$ were calculated by spectral deconvolution.

One approach to ensure continuous ion-release is to incorporate metallic elements into the silicate network. The $\mathrm{SiO}_{4}$ tetrahedron is the basic building unit of the silicate glasses and the tetrahedron can bond to each other through $\mathrm{Si}-\mathrm{O}-\mathrm{Si}$ bonds. One pulse ${ }^{29} \mathrm{Si}$ MAS NMR measurement with high powder decoupling techniques is a most useful method to study the changes that occur in the local structure of silicate glasses during the incorporation of calcium into the silicate network, it is known that the introduction of calcium could break $\mathrm{Si}-\mathrm{O}-\mathrm{Si}$ bonds and causes the reduction of $\mathrm{Q}^{4}$ species as well as the increase of $\mathrm{Q}^{3}$ or $\mathrm{Q}^{2}$ species. ${ }^{22,23}$ Fig. 8 shows the ${ }^{29} \mathrm{Si}$ MAS NMR spectra of BGN-30 and calcium-free silica particles, respectively. ${ }^{29} \mathrm{Si}$ NMR spectra of both samples exhibited a broad peak due to the line broadening effect in solid-state NMR spectroscopy caused by the amorphous nature of silicate glasses. This broad peak is a combination of two peaks at $c a$. $-111 \mathrm{ppm}$ and -106 ppm corresponding to the $\mathrm{Q}^{4}$ and $\mathrm{Q}^{3}$ species, respectively. ${ }^{49}$ Thus the ${ }^{29} \mathrm{Si}$ MAS NMR spectra of both samples were simulated by two components for the quantitative evaluation of $\mathrm{Q}^{4}$ and $\mathrm{Q}^{3}$ species (see S-Fig. 1a and $\mathrm{b} \dagger$ ). The results show that BGN-30 amounted to $\sim 15 \%$ of $\mathrm{Q}^{3}$ species while calcium-free sample contained $\sim 10 \%$ of $Q^{3}$ species, respectively (Fig. 8). The higher $\mathrm{Q}^{3}$ amount in BGN-30 is likely due to the breaking up of $\mathrm{Si}-\mathrm{O}-\mathrm{Si}$ bonds caused by the incorporation of calcium into the silicate network. Thus, combined with the fact that BGN-30 could release $\mathrm{Ca}^{2+}$ ions stably in DMEM for up to 14 days, it could be concluded that a certain amount of calcium indeed entered the silicate network in BGN-30.

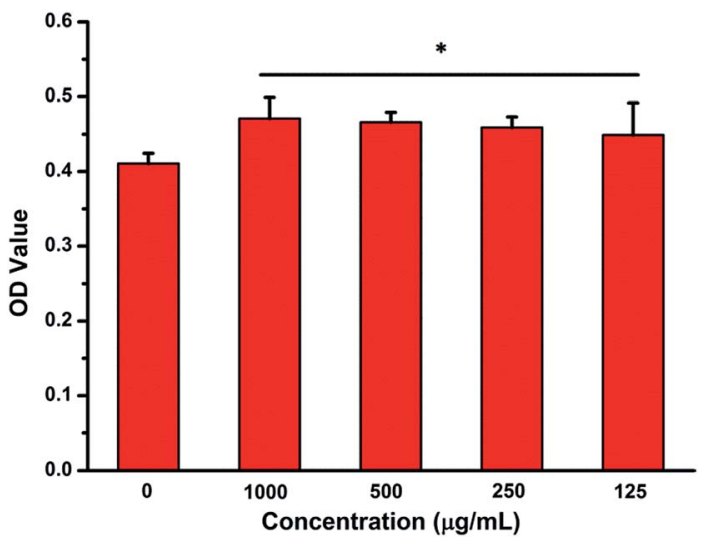

Fig. 9 MTT results of $B G N-30$ extracts at different concentration towards rBMSCs after culture for $24 \mathrm{~h}$. ${ }^{*} P<0.05$, cells cultured in LDMEM were set as the control group.

3.4.3. In vitro cytotoxicity. Fig. 9 shows the MTT results of BGN-30 extracts after culture with rBMSCs for $24 \mathrm{~h}$. Compared with the control, the extracts at all concentrations were noncytotoxic. No significant difference could be observed among the different concentrations, but a trend showing that BGN extracts enhance rBMSCs growth could be noted. This positive response may be attributed to the presence of released silicon and calcium in the medium. ${ }^{24}$

Based on the above results, it could be concluded that the monodispersed BGN-30 are bioactive and non-cytotoxic. In addition, they can release silicon and calcium ions in a stable and continuous manner. These features make BGN-30 promising materials for bone regeneration applications.

\section{Conclusions}

In this study, the influence of timing of calcium nitrate addition during BGN preparation on the resulting BGN properties was investigated. The results showed that the addition timing can affect the obtained BGN from the aspects of morphology, dispersity and composition. With delayed addition of calcium nitrate, more regular, homogenous and better dispersed BGN could be obtained. In addition, the gap between the nominal and the actual compositions widened with the delay of calcium nitrate addition. A facile and rapid synthetic routine for spherical monodispersed BGN was developed. This kind of BGN could be synthesized by adding calcium nitrate more than 30 min after mixing solutions A and B under the experimental condition of this study. Furthermore, BGN-30 showed bioactivity and continuous ion-release capability in DMEM, as well as no cytotoxic effects on rBMSCs. Therefore, the obtained monodispersed BGN are promising materials for biomedical applications, particularly for bone regeneration.

\section{Acknowledgements}

K. Z. acknowledges the fellowship from China Scholarship Council (CSC; No. 201206740003). M. H. and N. T. gratefully 
acknowledge financial support by the German Science Foundation (DFG) in the frame of its Cluster of Excellence "Engineering of Advanced Materials”. A. M. B. thanks to Talent-Hub Program funded by the Junta de Andalucía and the European Commission under the Co-funding of the 7th Framework Program in the People Program (Marie Curie Special Action). Authors also acknowledge the Laboratory for Nanoscopies and Spectroscopies (LANE) at the ICMS for TEM facilities.

\section{References}

1 J. R. Jones, Acta Biomater., 2013, 9, 4457-4486.

2 M. N. Rahaman, D. E. Day, B. Sonny Bal, Q. Fu, S. B. Jung, L. F. Bonewald and A. P. Tomsia, Acta Biomater., 2011, 7, 2355-2373.

3 H. Wang, S. Zhao, J. Zhou, Y. Shen, W. Huang, C. Zhang, M. N. Rahaman and D. Wang, J. Mater. Chem. B, 2014, 2, 8547-8557.

4 K. Zheng, Z. Wu, J. Wei, C. Rüssel, W. Liang and A. R. Boccaccini, J. Mater. Sci.: Mater. Med., 2015, 26, 224234.

5 Q. Chen, R. P. Garcia, M. Josemari, U. Pérez de Larraya, N. Garmendia, Q. Yao and A. R. Boccaccini, ACS Appl. Mater. Interfaces, 2015, 7, 24715-24725.

6 T. J. Brunner, R. N. Grass and W. J. Stark, Chem. Commun., 2006, 1384-1386.

7 M. Erol-Taygun, K. Zheng and A. R. Boccaccini, Int. J. Appl. Glass Sci., 2013, 4, 136-148.

8 G. M. Luz, L. Boesel, A. del Campo and J. F. Mano, Langmuir, 2012, 28, 6970-6977.

9 A. Hoppe, B. Sarker, R. Detsch, N. Hild, D. Mohn, W. J. Stark and A. R. Boccaccini, J. Non-Cryst. Solids, 2014, 387, 41-46.

10 Y. Xue, Y. Du, J. Yan, Z. Liu, P. X. Ma, X. Chen and B. Lei, J. Mater. Chem. B, 2015, 3, 3831-3839.

11 D. S. Couto, Z. Hong and J. F. Mano, Acta Biomater., 2009, 5, 115-123.

12 M. Sohrabi, S. Hesaraki, A. Kazemzadeh and M. Alizadeh, Mater. Sci. Eng., C, 2013, 33, 3730-3744.

13 A. R. Boccaccini, M. Erol, W. J. Stark, D. Mohn, Z. Hong and J. F. Mano, Compos. Sci. Technol., 2010, 70, 1764-1776.

14 W. Xia and J. Chang, Mater. Lett., 2007, 61, 3251-3253.

15 G. Miao, X. Chen, H. Dong, L. Fang, C. Mao, Y. Li, Z. Li and Q. Hu, Mater. Sci. Eng., C, 2013, 33, 4236-4243.

16 B. Lei, K.-H. Shin, Y.-W. Moon, D.-Y. Noh, Y.-H. Koh, Y. Jin and H.-E. Kim, J. Am. Ceram. Soc., 2012, 95, 30-33.

17 K. Zheng, J. A. Bortuzzo, Y. Liu, W. Li, M. Pischetsrieder, J. Roether, M. Lu and A. R. Boccaccini, Colloids Surf., B, 2015, 135, 825-832.

18 D. Santhiya, H. K. Alajangi, F. Anjum, S. Murugavel and M. Ganguli, J. Mater. Chem. B, 2013, 1, 6329-6338.

19 O. Tsigkou, S. Labbaf, M. M. Stevens, A. E. Porter and J. R. Jones, Adv. Healthcare Mater., 2014, 3, 115-125.

20 A. Lukowiak, J. Lao, J. Lacroix and J.-M. Nedelec, Chem. Commun., 2013, 49, 6620-6622.

21 Q. Hu, Y. Li, G. Miao, N. Zhao and X. Chen, RSC Adv., 2014, 4, 22678-22687.
22 C. J. Brinker, Sol-Gel Science: The Physics and Chemistry of Sol-Gel Processing, Academic Press, 1990.

23 R. K. Iler, The chemistry of silica: solubility, polymerization, colloid and surface properties, and biochemistry, WileyInterscience, 1979.

24 A. Hoppe, N. S. Güldal and A. R. Boccaccini, Biomaterials, 2011, 32, 2757-2774.

25 L. L. Hench, J. Mater. Sci.: Mater. Med., 2006, 17, 967-978.

26 P. J. Marie, Bone, 2010, 46, 571-576.

27 G. Poologasundarampillai, B. Yu, O. Tsigkou, D. Wang,

F. Romer, V. Bhakhri, F. Giuliani, M. M. Stevens, D. S. McPhail, M. E. Smith, J. V. Hanna and J. R. Jones, Chem.-Eur. J., 2014, 20, 8149-8160.

28 S. Lin, C. Ionescu, K. J. Pike, M. E. Smith and J. R. Jones, J. Mater. Chem., 2009, 19, 1276-1282.

29 S. Labbaf, O. Tsigkou, K. H. Müller, M. M. Stevens, A. E. Porter and J. R. Jones, Biomaterials, 2011, 32, 10101018.

30 A.-C. J. H. Johnson, P. Greenwood, M. Hagström, Z. Abbas and S. Wall, Langmuir, 2008, 24, 12798-12806.

31 A. A. R. de Oliveira, D. A. de Souza, L. L. S. Dias, S. M. de Carvalho, H. S. Mansur and M. de Magalhães Pereira, Biomed. Mater., 2013, 8, 025011.

32 C. Wu, W. Fan and J. Chang, J. Mater. Chem. B, 2013, 1, 27102718.

33 A. Ramila, F. Balas and M. Vallet-Regi, Chem. Mater., 2002, 14, 542-548.

34 X. Li, L. Zhang, X. Dong, J. Liang and J. Shi, Microporous Mesoporous Mater., 2007, 102, 151-158.

35 K. Zheng, A. Solodovnyk, W. Li, O.-M. Goudouri, C. Stähli, S. N. Nazhat and A. R. Boccaccini, J. Am. Ceram. Soc., 2015, 98, 30-38.

36 M. Peter, N. S. Binulal, S. V. Nair, N. Selvamurugan, H. Tamura and R. Jayakumar, Chem. Eng. J., 2010, 158, 353-361.

37 Z. Hong, G. M. Luz, P. J. Hampel, M. Jin, A. Liu, X. Chen and J. F. Mano, J. Biomed. Mater. Res., Part A, 2010, 95, 747-754.

38 A. R. Curtis, N. X. West and B. Su, Acta Biomater., 2010, 6, 3740-3746.

39 Q. Hu, X. Chen, N. Zhao and Y. Li, Mater. Lett., 2013, 106, 452-455.

40 Y. Fan, P. Yang, S. Huang, J. Jiang, H. Lian and J. Lin, J. Phys. Chem. C, 2009, 113, 7826-7830.

41 H. Aguiar, J. Serra, P. González and B. León, J. Non-Cryst. Solids, 2009, 355, 475-480.

42 M. Cerruti, V. Bolis, G. Magnacca and C. Morterra, Phys. Chem. Chem. Phys., 2004, 6, 2468-2479.

43 Y. Zhu, C. Wu, Y. Ramaswamy, E. Kockrick, P. Simon, S. Kaskel and H. Zreiqat, Microporous Mesoporous Mater., 2008, 112, 494-503.

44 P. Saravanapavan and L. L. Hench, J. Non-Cryst. Solids, 2003, 318, 1-13.

45 W. Li, P. Nooeaid, J. a. Roether, D. W. Schubert and A. R. Boccaccini, J. Eur. Ceram. Soc., 2014, 34, 505-514.

46 M. Mačković, A. Hoppe, R. Detsch, D. Mohn, W. J. Stark, E. Spiecker and A. R. Boccaccini, J. Nanopart. Res., 2012, 14, 966-988. 
47 R. Li, A. E. Clark and L. L. Hench, J. Appl. Biomater., 1991, 2, 231-239.

48 C. Wang, Y. Xie, A. Li, H. Shen, D. Wu and D. Qiu, ACS Appl. Mater. Interfaces, 2014, 6, 4935-4939.

49 S. Chen, A. Osaka, S. Hayakawa, K. Tsuru, E. Fujii and K. Kawabata, J. Sol-Gel Sci. Technol., 2008, 48, 322-335.
50 C. Stähli, M. James-Bhasin, A. Hoppe, A. R. Boccaccini and S. N. Nazhat, Acta Biomater., 2015, 19, 15-22.

51 D. Rohanová, A. R. Boccaccini, D. Horkavcová, P. Bozděchová, P. Bezdička and M. Častorálová, J. Mater. Chem. B, 2014, 2, 5068-5076. 